\title{
A GENERALIZED DOMAIN FOR SEMIGROUP GENERATORS
}

\author{
MICHAEL G. CRANDALL ${ }^{1}$
}

\begin{abstract}
A generalized domain $\hat{D}(A)$ is assigned to a certain class of generators $A$ of semigroups of nonlinear transformations $S$ on Banach spaces. $\hat{D}(A)$ is then characterized in two ways. $\hat{D}(A)$ is the set of $x$ such that $S(t) x$ is locally Lipschitz continuous in $t$ or, equivalently, the set of $x$ which can lie in the domain of suitable extensions of $A$.
\end{abstract}

Let $X$ be a Banach space, $C$ be a subset of $X, \omega$ be a real number and $S \in Q_{\omega}(C)$, i.e. $S(t): C \rightarrow C$ for $t \geqq 0, S(t) S(\tau)=S(t+\tau)$ for $t, \tau \geqq 0, e^{\omega t}$ is a Lipschitz constant for $S(t)$ and $S(t) x$ is continuous in $t$ for $x \in C$. Assume $S$ is generated by a set $-A$, that is

$$
S(t) x=\lim _{n \rightarrow \infty}(I+(t / n) A)^{-n} x \text { for } t>0 \text { and } x \in C
$$

and $A+\omega I$ is accretive (see [2] or [4] for undefined terms as used here). In general, $S(t)$ will not leave $D(A)$ invariant and $S(t) x$ can be nowhere differentiable in $t$ even if $x \in D(A)$. These phenomena do not indicate a weakness of the theory of nonlinear semigroups. Rather, they reflect its generality. Indeed, there are Cauchy problems for nonlinear partial differential equations which exhibit similar behaviour and which fall within the scope of the abstract semigroup theory.

In this note we assign a generalized domain $\hat{D}(A)$ to each set $A$ such that $A+\omega I$ is accretive and $R(I+\lambda A) \supset \mathrm{Cl}(D(A)$ ) (where $\mathrm{Cl}$ denotes closure) for sufficiently small positive $\lambda$. It is shown that if (1) holds, then $\hat{D}(A) \cap C$ is precisely the set of those $x \in C$ for which $S(t) x$ is Lipschitz continuous in $t$ on compact subsets of $[0, \infty)$. It follows that $\hat{D}(A) \cap C$ is invariant under $S(t)$. Simple examples show $\hat{D}(A)$ need not equal $D(A)$ even if $A$ is linear and densely defined.

If $X$ is reflexive, then $A$ has an extension $B$ such that $B+\omega I$ is accretive and $\hat{D}(B)=\hat{D}(A)=D(B)$, and most of our results are known. See [11]. Interest centers in the nonreflexive case here. Examples of Cauchy problems in nonreflexive settings may be found in [2], [3], [9] and [10].

Received by the editors December 17, 1971.

AMS (MOS) subject classifications (1970). Primary 47H15; Secondary 34G05.

${ }^{1}$ Sponsored by the U.S. Army under Contract. No. DA-31-124-ARO-D-462. 
1. Definition and characterization of $\hat{D}(A)$. If $A \subseteq X \times X$ and $\lambda$ is a nonzero real number, we let $D_{\lambda}=R(I+\lambda A), J_{\lambda}=(I+\lambda A)^{-1}, A_{\lambda}=$ $\lambda^{-1}\left(I-J_{\lambda}\right) . \mathscr{A}(\omega)$ denotes the set of subsets of $X \times X$ such that $A+\omega I$ is accretive. When necessary, we write $\mathscr{A}(\omega, X)$ to display the space $X$.

Denote the norm in $X$ by \|\| . We need the following simple facts.

Lemma 1. Let $A \in \mathscr{A}(\omega), \lambda>0, \lambda \omega<1$. Then the following statements hold:

(i) $J_{\lambda}$ is a function and $\left\|J_{\lambda} x-J_{\lambda} y\right\| \leqq(1-\lambda \omega)^{-1}\|x-y\|$ for $x, y \in D_{\lambda}$.

(ii) If $\lambda \geqq \mu>0$ and $x \in D_{\lambda} \cap D_{\mu}$, then $(1-\lambda \omega)\left\|A_{\lambda} x\right\| \leqq(1-\mu \omega)\left\|A_{\mu} x\right\|$.

(iii) If $x \in D_{\lambda} \cap D(A)$ and $y \in A x$, then $(1-\lambda \omega)\left\|A_{\lambda} x\right\| \leqq\|y\|$.

For a proof of (i) and (iii) above see [4, Lemma 1.2]. The monotonicity (ii) is observed in [6] in a special case. A proof is given in [7, Lemma 1.2].

Definition 1. Let $A \in \mathscr{A}(\omega)$ and $\mathscr{D}=\bigcup_{\kappa>0} \bigcap_{0<\lambda<\kappa} D_{\lambda}$. If $x \in \mathscr{D}$, then $|A x|=\lim _{\lambda \downarrow 0}\left\|A_{\lambda} x\right\|$. If $\mathscr{D} \supseteq D(A)$, then $\hat{D}(A)=\{x: x \in \mathscr{D}$ and $|A x|<\infty\}$.

Lemma 1 (ii) guarantees that $|A x|$ is defined for $x \in \mathscr{D}$.

Lemma 2. Let $A \in \mathscr{A}(\omega)$ and $\mathscr{D} \supset D(A)$. Then

$$
|A x| \leqq \inf \{\|y\|: y \in A x\} \text { for } x \in D(A)
$$

and $\mathrm{Cl}(D(A)) \supset \hat{D}(A) \supset D(A)$. Moreover, the map $x \rightarrow|A x|$ is lower semicontinuous on $\bigcap_{0<\lambda<\kappa} D_{\lambda}$ for each $\kappa>0$.

Proof. The inequality (1.1) follows at once from Lemma 1.1 (iii), and $\hat{D}(A) \supset D(A)$ follows from (1.1). The inclusion $\mathrm{Cl}(D(A)) \supset \hat{D}(A)$ follows from the definitions and the fact that $D(A)$ is the range of $J_{\lambda}$. The lower semicontinuity of $|A x|$ on $\bigcap_{0<\lambda<\kappa} D_{\lambda}$ follows from the Lipschitz continuity of $A_{\lambda}$ (a consequence of Lemma 1 (i) and the definition of $A_{\lambda}$ ) and the relation

$$
|A x|=\sup _{0<\lambda<\kappa}(1-\lambda \omega)\left\|A_{\lambda} x\right\| \quad(\text { for } \kappa \omega<1) .
$$

REMARK 1. The number on the right in (1.1) was denoted by $|A x|$ in [4]. All inequalities of [4] remain correct if $|A x|$ is understood as in Definition 1. See below.

THEOREM 1. Let $A \in \mathscr{A}(\omega), \lambda_{0}>0$ and $D_{\lambda} \supset \mathrm{Cl}(D(A))$ for $0<\lambda<\lambda_{0}$. Let $S$ be the semigroup on $\mathrm{Cl}(D(A))$ generated by $-A$ (i.e., $S \in Q_{\omega}(\mathrm{Cl}(D(A))$ is defined by (1)). Let

$$
L(x)=\liminf _{h ! 0} \frac{\|S(h) x-x\|}{h}
$$

for $x \in \mathrm{Cl}(D(A))$. Then $L(x)=|A x|$ for $x \in \mathrm{Cl}(D(A))$.

Proof. The existence of $S$ satisfying (1) is established in Theorem I of [4]. We first show $L(x) \leqq|A x|$. Indeed, if $x \in \mathrm{Cl}(D(A))$ and $t>0$, 
Lemma 1 and the definitions yield

$$
\begin{aligned}
\|S(t) x-x\| & =\lim _{n \rightarrow \infty}\left\|J_{t / n}^{n} x-x\right\| \leqq \underset{n \rightarrow \infty}{\lim \sup } \sum_{k=1}^{n}\left\|J_{t / n}^{k} x-J_{t / n}^{k-1} x\right\| \\
& \leqq \limsup _{n \rightarrow \infty} \sum_{k=1}^{n}\left(1-\frac{t}{n} \omega\right)^{-k+1}\left\|J_{t / n} x-x\right\| \\
& \leqq t \limsup _{n \rightarrow \infty} \frac{1}{n} \sum_{k=1}^{n}\left(1-\frac{t}{n} \omega\right)^{-k+1}\left\|A_{t / n} x\right\| \\
& \leqq t|A x| \lim _{n \rightarrow \infty} \frac{1}{n} \sum_{k=1}^{n}\left(1-\frac{t}{n} \omega\right)^{-k}=t \frac{e^{\omega t}-1}{\omega t}|A x|
\end{aligned}
$$

where we set $\left(e^{\omega t}-1\right) / \omega=t$ if $\omega=0$. It follows that $L(x) \leqq|A x|$. The inequality $|A x| \leqq L(x)$ follows from the fact that if $\left[x_{0}, y_{0}\right] \in A, x \in$ $\mathrm{Cl}(D(A))$, and $z^{*} \in F\left(x-x_{0}\right)$, then

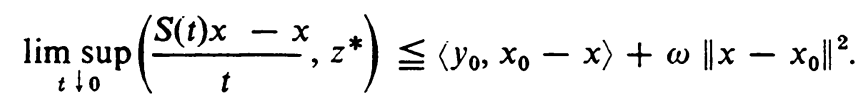

Here we assume, without loss of generality, that $X$ is a real Banach space. The value of $z^{*} \in X^{*}$ at $z \in X$ is denoted by $\left(z, z^{*}\right)$. If $z \in X, F(z)=$ $\left\{z^{*} \in X^{*}:\left(z, z^{*}\right)=\|z\|^{2}=\left\|z^{*}\right\|^{2}\right\}$. The function $\langle$,$\rangle appearing in (1.2) is$ defined by

$$
\langle y, x\rangle=\max \left\{\left(y, x^{*}\right): x^{*} \in F(x)\right\} \text { for } x, y \in X .
$$

The inequality (1.2) is obtained in [4, Lemma 2.9] under the technical assumption that $D_{\lambda}$ contained the convex hull of $D(A)$. Miyadera removed this restriction in [11] and gave a proof for $\omega=0$. A more general result is obtained in the proof of Theorem 3.2 of [7]. Clearly (1.2) implies that if $\left[x_{0}, y_{0}\right] \in A$ then

$$
-L(x)\left\|x-x_{0}\right\| \leqq\left\langle y_{0}, x_{0}-x\right\rangle+\omega\left\|x_{0}-x\right\|^{2} .
$$

Choose $x_{0}=J_{\lambda} x, y_{0}=A_{\lambda} x_{0}$. Then $x-x_{0}=\lambda y_{0}=\lambda A_{\lambda} x$ and (1.4) becomes, upon dividing by $\lambda$,

$$
-L(x)\left\|A_{\lambda} x\right\| \leqq-\left\|A_{\lambda} x\right\|^{2}+\omega \lambda\left\|A_{\lambda} x\right\|^{2} .
$$

Letting $\lambda \downarrow 0$, we find $|A x| \leqq L(x)$. The proof is complete.

COROLlaRY 1. Let the assumptions of Theorem 1 hold. Then $\hat{D}(A)=$ $\{x: x \in \mathrm{Cl}(D(A))$ and $S(t) x$ is Lipschitz continuous in $t$ on bounded subsets of $[0, \infty)\}$. Moreover, $S(t): \hat{D}(A) \rightarrow \hat{D}(A)$ for each $t \geqq 0$.

Corollary 1 is an immediate consequence of Theorem 1 and the following simple lemma. 
Lemma 3. Let $C \subseteq X$ and $S \in Q_{\omega}(C)$. Then, for each $x \in C$ and $t, \tau \geqq 0$,

$$
\|S(t+\tau) x-S(t) x\| \leqq e^{\omega t}\left(\left(e^{\omega \tau}-1\right) / \omega\right) L(x)
$$

where $L(x)$ is defined as in Theorem 1 .

Proof. We sketch the proof. Also see, e.g., [5, Lemma 1.1]. Since $S \in Q_{\omega}(C)$ is suffices to show (1.5) for $t=0$ and $L(x)<\infty$. Let $K>L(x)$. Then there is a sequence $\left\{t_{k}\right\}$ of positive numbers convergent to zero such that

$$
\left\|S\left(t_{k}\right) x-x\right\| \leqq K t_{k}, \quad k=1,2, \cdots .
$$

Let $\left\{n_{k}\right\}$ be a sequence of positive integers such that $n_{k} t_{k} \rightarrow \tau$. Then $S \in$ $Q_{\omega}(C)$ and (1.6) give

$$
\begin{aligned}
\|S(\tau) x-x\| & =\lim _{k \rightarrow \infty}\left\|S\left(n_{k} t_{k}\right) x-x\right\| \\
& \leqq \limsup _{k \rightarrow \infty} \sum_{j=1}^{n_{k}}\left\|S\left(j t_{k}\right) x-S\left((j-1) t_{k}\right) x\right\| \\
& \leqq \lim _{k \rightarrow \infty} K \sum_{j=1}^{n_{k}} e^{(j-1) t_{k} \omega} t_{k}=K\left(\frac{e^{\omega \tau}-1}{\omega}\right) .
\end{aligned}
$$

Since $K>L(x)$ was arbitrary, the proof is complete.

REMARK 2. It follows from Lemma 3 that

$$
f(t)=\lim _{\tau \downarrow 0} \frac{\|S(t+\tau) x-S(t) x\|}{\tau}
$$

exists for $t \geqq 0$ and $e^{-\omega t} f(t)$ is nonincreasing in $t(f(t)=\infty$ is allowed here).

The next result gives a characterization of $\hat{D}(A)$ independent of the semigroup theory.

TheOREM 2. Let $A \in \mathscr{A}(\omega, X)$ and the set $\mathscr{D}$ of Definition 1 include $\mathrm{Cl}(D(A))$. Then $x \in \hat{D}(A)$ if and only if there is an element $y^{* *}$ of the second dual $X^{* *}$ of $X$ such that

$$
A \cup\left\{\left[x, y^{* *}\right]\right\} \in \mathscr{A}\left(\omega, X^{* *}\right) .
$$

If $x \in \hat{D}(A)$, the $y^{* *}$ above can be chosen so that $|A x|=\left\|y^{* *}\right\|$.

Proof. In the statement and proof of the theorem, $X$ is regarded as a subspace of $X^{* *}$ via the canonical imbedding. One direction is trivial. If $x \in \mathrm{Cl}(D(A)), y^{* *} \in X^{* *}$ and $B=A \cup\left\{\left[x, y^{* *}\right]\right\} \in \mathscr{A}\left(\omega, X^{* *}\right)$, then clearly $|A x|=|B x| \leqq\left\|y^{* *}\right\|<\infty$, and $x \in \hat{D}(A)$.

To establish the opposite assertion, let $x \in \mathrm{Cl}(D(A))$ and $|A x|<\infty$. It is known that $A \in \mathscr{A}(\omega)$ is equivalent to the condition that

$$
\left\langle y_{1}-y_{2}, x_{1}-x_{2}\right\rangle \geqq-\omega\left\|x_{1}-x_{2}\right\|^{2} \text { for }\left[x_{i}, y_{i}\right] \in A, i=1,2 \text {, }
$$


where $\langle$,$\rangle is defined in (1.3). (See [8].) Let \left[x_{0}, y_{0}\right]$ be an arbitrary element of $A$. Since $\left[J_{\lambda} x, A_{\lambda} x\right] \in A,(1.8)$ implies

$$
\begin{aligned}
\left\langle A_{\lambda} x, J_{\lambda} x-x_{0}\right\rangle+\left\langle-y_{0}, J_{\lambda} x-x_{0}\right\rangle & \geqq\left\langle A_{\lambda} x-y_{0}, J_{\lambda} x-x_{0}\right\rangle \\
& \geqq-\omega\left\|x_{0}-J_{\lambda} x\right\|^{2} .
\end{aligned}
$$

Notice that $\left(u-v, u^{*}-v^{*}\right) \geqq 0$ whenever $u^{*} \in F(u), \quad v^{*} \in F(v)$ (i.e. $F: X \rightarrow 2^{X^{*}}$ is monotone). This implies

$$
\begin{aligned}
\left(A_{\lambda} x, z^{*}\right) & \geqq\left\langle A_{\lambda} x,\left(x-x_{0}\right)-\lambda_{\lambda} x\right\rangle \\
& =\left\langle A_{\lambda} x, J_{\lambda} x-x_{0}\right\rangle \text { for } z^{*} \in F\left(x-x_{0}\right) .
\end{aligned}
$$

Together, (1.9) and (1.10) imply

$$
\begin{array}{r}
\left(A_{\lambda} x, z^{*}\right)+\left\langle-y_{0}, J_{\lambda} x-x_{0}\right\rangle \geqq-\omega\left\|x_{0}-J_{\lambda} x\right\|^{2} \\
\text { for } z^{*} \in F\left(x-x_{0}\right) .
\end{array}
$$

Let $y^{* *}$ be a cluster point of $A_{\lambda} x$ in the weak-star topology on $X^{* *}$ as $\lambda \downarrow 0$. Since $\left\|J_{\lambda} x-x\right\| \rightarrow 0$ as $\lambda \downarrow 0$ and $\langle$,$\rangle is upper semicontinuous (see,$ e.g., [4, Lemma 2.16]), passing to the limit inferior as $\lambda \downarrow 0$ in (1.11) yields

$$
\left(y^{* *}, z^{*}\right)+\left\langle-y_{0}, x-x_{0}\right\rangle \geqq-\omega\left\|x_{0}-x\right\|^{2}
$$

for all $z^{*} \in F\left(x-x_{0}\right)$. (Here $\left(y^{* *}, z^{*}\right)$ is the value of $y^{* *}$ at $z^{*}$.) Choose an element $z^{*}$ of $F\left(x-x_{0}\right)$ such that $\left\langle-y_{0}, x-x_{0}\right\rangle=-\left(y_{0}, z^{*}\right)$. With this choice (1.12) yields

$$
\left\langle y^{* *}-y_{0}, x-x_{0}\right\rangle \geqq\left(y^{* *}-y_{0}, z^{*}\right) \geqq-\omega\left\|x-x_{0}\right\|^{2} .
$$

Since $\left[x_{0}, y_{0}\right] \in A$ was arbitrary, it follows that $A \cup\left\{\left[x, y^{* *}\right]\right\} \in \mathscr{A}\left(\omega, X^{* *}\right)$. Clearly, $\left\|y^{* *}\right\| \leqq|A x| \leqq\left\|y^{* *}\right\|$, and the proof is complete.

REMARK 3. The inequality (1.13) involves $F\left(x-x_{0}\right)$ as a subset of $X^{*}$. The corresponding inequality with $F\left(x-x_{0}\right)$ as a subset of $X^{* * *}$ is weaker. We have found no useful consequences of this observation.

REMARK 4. It also follows from (1.2) that if $y^{* *}$ is a weak-star cluster point of $t^{-1}(x-S(t) x)$ as $t \downarrow 0$, then $A \cup\left\{\left[x, y^{* *}\right]\right\} \in \mathscr{A}\left(\omega, X^{* *}\right)$.

2. Examples. If we set $X=C_{0}([0, \infty)$ ) (real-valued continuous functions on $[0, \infty)$ tending to zero at $\infty$, under the maximum norm) and

$$
A f=-f^{\prime}, \quad D(A)=\left\{f \in X ; f^{\prime} \in X\right\},
$$

where $f^{\prime}$ denotes the derivative of $f$. The corresponding semigroup $S$ is translations, i.e.

$$
S(t) f(x)=f(x+t) \text { for } x, t \in[0, \infty), f \in X .
$$


Here

$$
A_{\lambda} f(x)=\frac{1}{\lambda^{2}} \int_{x}^{\infty} \exp ((x-s) / \lambda)(f(x)-f(s)) d s .
$$

Using Theorem 1 and (2.2) we see that $\hat{D}(A)=\{f \in X: f$ is Lipschitz continuous $\}$ and $|A f|$ is just the least Lipschitz constant for $f$. This information is harder to extract from (2.3). The main point, however, is that $\hat{D}(A)$ is strictly larger than $D(A)$ in this case. Theorem 2 can be illustrated in this simple case as well. Regarding bounded Borel measurable functions as a subset of $X^{* *}$ in the natural way, set

$$
f^{* *}(x)=\underset{n \rightarrow \infty}{\liminf } n(f(x+1 / n)-f(x)) \text { for } f \in \hat{D}(A) .
$$

Then $A \cup\left[f,-f^{* *}\right] \in \mathscr{A}\left(0, X^{* *}\right)$.

Next we show $\hat{D}$ is invariant under certain perturbations and apply this to an example of Webb [12].

THEOREM 3. Let $A \in \mathscr{A}(\omega)$ and $B$ be a continuous map of $\mathrm{Cl}(D(A))$ into $X$. Assume further that $T=A+B \in \mathscr{A}(\omega)$ and

$$
R(I+\lambda T) \cap R(I+\lambda A) \supseteq \mathrm{Cl}(D(A))=\mathrm{Cl}(D(T))
$$

for $0<\lambda<\lambda_{0}$, where $\lambda_{0}$ is a positive number. Then $\hat{D}(T)=\hat{D}(A)$.

Proof. Let $x \in \mathrm{Cl}(D(A))$ and $\left[x_{\lambda}, y_{\lambda}\right] \in A$ satisfy

$$
x_{\lambda}+\lambda\left(y_{\lambda}+B x_{\dot{\lambda}}\right)=x
$$

for $0<\lambda<\lambda_{0}$. Then $\lim _{\lambda \downarrow 0} x_{\lambda}=x$ and so $\lim _{i ! 0}\left\|B x_{i}-B x\right\|=0$. Hence

$$
\lim _{i \downarrow 0}\left\|T_{i} x\right\|=\lim _{i \downarrow 0}\left\|y_{\lambda}+B x_{i}\right\|
$$

is finite if and only if

if finite. Now

$$
\lim _{\lambda \downarrow 0}\left\|y_{\lambda}\right\|=\lim _{\lambda \downarrow 0}\left\|A_{\lambda}\left(x-\lambda B x_{\lambda}\right)\right\|
$$

$$
\left\|y_{\lambda}-A_{\lambda} x\right\|=\left\|A_{\lambda_{\lambda}}\left(x-\lambda B x_{\lambda}\right)-A_{\lambda} x\right\| \leqq\left(1+(1-\lambda \omega)^{-1}\right)\left\|B x_{\lambda}\right\|
$$

by Lemma 1 (iii) and the definition of $A_{\lambda}$. It follows at once that $|A x|<\infty$ if and only if $|T x|<\infty$. The proof is complete.

Webb [12] proved that if $-A$ is the infinitesimal generator of a strongly continuous semigroup of linear contractions on $X$ and $B: X \rightarrow X$ is continuous and accretive, then $R(I+\lambda(A+B))=X$ for $\lambda>0$. He observed that if we take $X=C_{0}([0, \infty)), A f=-f^{\prime}$ as before, and $B f=\max \{f, 0\}$, then the semigroup generated by $-(A+B)$ does not leave $D(A)$ invariant. It 
follows from Theorem 3 and Corollary 1 that $\hat{D}(A+B)=\hat{D}(A)$ is invariant. This remains true if we let $B f(x)=g(f(x))$ where $g: R \rightarrow R$ is any continuous monotonically increasing function such that $g(0)=0$. Theorem 3 generalizes easily to cases in which $B$ is only required to satisfy local estimates of the form $\|B x\| \leqq k|A x|+K$ where $k<1$. In particular, the analogue of Theorem 3 for the situation of Lemma 1 of [1] holds. There seem to be no general results concerning $R(I+\lambda(A+B))$ in nonreflexive spaces $X$. We mention that the hypothesis of linearity of $A$ in Webb's result may be dropped if $B$ is assumed to be locally uniformly continuous.

ADDED IN PROOF. U. Westphal has kindly informed the author that the set called $\hat{D}(A)$ here is well known in linear theory. See the references of Westphal's note, Sur la saturation pour des semi-groupes non linéaires, C.R. Acad. Sci. Paris 274 (1972), 1351-1353, which is closely related to this paper.

\section{REFERENCES}

1. B. Calvert and K. Gustafson, Multiplicative perturbation of nonlinear m-accretive operators, J. Functional Analysis 10 (1972), 149-157.

2. M. G. Crandall, Semigroups of nonlinear transformations in Banach spaces, Contributions to Nonlinear Functional Analysis, Eduardo H. Zarantonello (Editor), Academic Press, New York, 1971, pp. 157-179.

3. - The semigroup approach to first order quasilinear equations in several space variables, Israel J. Math. (to appear).

4. M. G. Crandall and T. M. Liggett, Generation of semi-groups of nonlinear transformations on general Banach spaces, Amer. J. Math. 93 (1971), 265-298.

5. M. G. Crandall and A. Pazy, Semi-groups of nonlinear contractions and dissipative sets, J. Functional Analysis 3 (1969), 376-418. MR 39 \#4705.

6. - On accretive sets in Banach spaces, J. Functional Analysis 5 (1970), 204-217. MR 41 \#4201.

7. - Nonlinear evolution equations in Banach spaces, Israel J. Math. 11 (1972), 57-94.

8. T. Kato, Nonlinear semigroups and evolution equations, J. Math. Soc. Japan 19 (1967), 508-520. MR 37 \#1820.

9. Y. Konishi, Some examples of nonlinear semi-groups in Banach lattices, J. Fac. Sci. Univ. Tokyo Sect. IA 18 (1972), 537-543.

10. - On $u_{t}=u_{x x}-F\left(u_{x}\right)$ and the differentiability of the nonlinear semi-group associated with it, Proc. Japan Acad. 48 (1972), 281-286.

11. I. Miyadera, Some remarks on semigroups of nonlinear operators, Tôhoku Math. J. 23 (1971), 245-258.

12. G. F. Webb, Continuous nonlinear perturbations of linear accretive operators in Banach spaces, J. Functional Analysis 10 (1972), 191-203.

Mathematics Research Center, University of Wisconsin, Madison, Wisconsin 53705

Department of Mathematics, University of California, los Angeles, CaliFORNIA 90024 